\title{
Performance Study of Queuing Delay in CRN Using Different Proposed Spectrum Handoff Approaches
}

\author{
Shimaa Ali ${ }^{1}$, Mona Shokair ${ }^{2}$, Moawad I. Dessouky ${ }^{3}$, Nagy Messiha $^{4}$ \\ ${ }^{1234}$ Dept. of Electrical Engineering, Faculty of Engineering, Menoufia University \\ (Received: 24 Aug. 2017 - Accepted: 12 Oct. 2017)
}

\section{Abstract}

Spectrum handoff in cognitive radio networks is to have a seamless switch between channels consequently, a high quality of service for the secondary users. This paper proposes three techniques for spectrum handoff. The first proposed one based on the low priority user leaves the channel to another channel. The second proposed model based on the low priority user stays on the same channel with resuming its transmission when the high priority user finished its transmission. Finally, the third proposed model based on the low priority user stays on the same channel with restarting its transmission when the high priority user finished its transmission. The obtained results indicate that the three proposed system models give different queuing delay performances for each user. The techniques of the models and the obtained results are important for evaluating the total system time, service time and queuing delay for secondary users. Consequently, the quality of service for the secondary users and hence the useful for cognitive radio network design and optimization will be obtained.

\section{Introduction}

Due to the scarcity of spectrum band in wireless communications, a new technique called Cognitive Radio Networks (CRN) had been proposed to allow the secondary users to use the spectrum bands, which are already licensed to the Primary User (PU), in an opportunistic manner. CRN was presented in order to enhance the efficiency of the spectrum resources utilization [1,2]. Spectrum handoff is one of the main procedures of CRNs. It 
means that during the transmission of the Secondary User (SU) if the PU returns to its channel, SU has to leave this channel and the priority is given to the PU. The purpose of spectrum handoff is to make a seamless switch over from channel to another to have a satisfied quality of service for the SU transmission [3].

Recently, there are many studies on the spectrum handoff issue. Proposed spectrum handoff techniques by using fuzzy logic control were presented in $[4,5]$. The target channel selection issue was discussed in [6 - 8]. According to the timing of determining the target channels, the handoff process can be classified into two kinds. The first one is called a proactive spectrum handoff and the other is called a reactive spectrum handoff [9-11]. In [12], a novel spectrum handoff strategy has proposed, aiming at reducing the unnecessary handoff operations while considering a delay bound requirement. A mixed preemptive and non-preemptive resume priority (PRP/NPRP) M/G/1 queuing model with prioritized transmissions based on delay-sensitive applications proposed in [13].

In this paper, three techniques are proposed on a system of one primary user and three secondary users. Change policy, stay policy with resuming the transmission which used Preemptive Resume Priority (PRP) M/ G/1 queuing model [14] and stays with restarting the transmission which used Preemptive Repeat Identical (PRI) M/ G/1 queuing model [15].

The remainder of the paper is organized as follows: Section 2 describes the queuing models. The proposed techniques will be discussed in Section 3. Section 4 presents the simulation results. Finally, Section 5 concludes the paper.

\section{Queuing Models:}

The proposed techniques applied M/G/1 queue model [14]. This queue model represents the length of a queue in a system with a single server where the arrival time distribution is Poisson distribution and the service time distribution is general. The second proposed technique used PRP M/G/1 queuing model [14]. The third proposed technique applied PRI M/G/1 queuing model [15].

\subsection{PRP M/G/1 Queuing Model:}


The features of PRP M/G/1 queuing model are [14]:

1. There are two types of users that may use the channel: the high priority user which is the primary user and the low priority user which is one of the secondary users.

2. The primary user has a preemptive priority to use the channel consequently; it can interrupt the transmission of the secondary users. The interrupted secondary user resumes its unfinished transmission instead of repeating its whole transmission from the beginning.

\subsection{PRI M/G/1 Queuing Model:}

The features of PRI M/G/1 queuing model are [15] are the same features for PRP but the difference is the interrupted secondary user repeats its transmission from the beginning instead of resuming its unfinished transmission.

\section{Proposed System Models:}

The following assumptions are made in the proposed system models:

1. The system model consists of one primary user and three secondary users.

2. Only one user can transmit its data on the channel simultaneously.

3. The three secondary users have three priorities i.e, the SU1 has the first priority, the SU2 has the second priority and SU3 has the third priority.

4. The priority is for real-time data.

The block diagrams of the proposed system models shown in Fig.1, Fig.2 and Fig.3 are constructed using Simulink Matlab version 10. These block diagrams consist of:

- Task token generator: which responsible for generating the entities.

- Application task combiner: accept entities from the input ports and output them through a single entity output port i.e, it merges paths.

- Priority based-task queue: Store entities in sorted sequence for an undetermined length of time. The queue sorts entities according to ascending order.

- Task queue length scope: plot how the length of a queue changes over time. 
- Priority scope: plot the priority versus the simulation time.

- Channel: it likes a single server that serves one entity for a period of time and then outputs the entity through the OUT port. If the OUT port is blocked then the entity stays in this block until the port becomes unblocked. When the block permits preemption, an entity in the channel can depart early via the $\mathrm{P}$ port.

- Task complete block: measure the total system time and the service time then compute the queuing delay and plot it.

- Entity sink: terminate the path of the preempted entity. This block exists only in the change policy proposed model.

\subsection{The first proposed model:}

The first proposed system model is the change policy model is shown in Fig. 1. In this policy, the secondary user changes its transmission to another channel when the PU arrives and reclaims its channel.

\section{Proposed approach operation steps:}

- The tasks for one primary user and three secondary users represented as (PU, SU1, SU2, and SU3) are generated from the task token generators.

- The entities of the four users enter to the application task combiner block which merges the four paths and output them through a single output port which input to the priority based task queue block.

- The priority-based task queue block stores entities in specified sequence for an undetermined time. The capacity of the queue, which is the number of entities can be held in the queue, is assumed to be infinity. This block has two outputs: the first output is the task queue length and the second output is to show the priority value for each task through the priority scope.

- The tokens are passed to channel for task execution. The channel serves the user for a period of time. When the higher priority user arrives, the low priority users depart and change its channel before it completes its transmission via $\mathrm{P}$ port which input to the entity sink to leave the channel. The output of the channel input to the task complete block to compute the queuing delay for each user and plot it. 


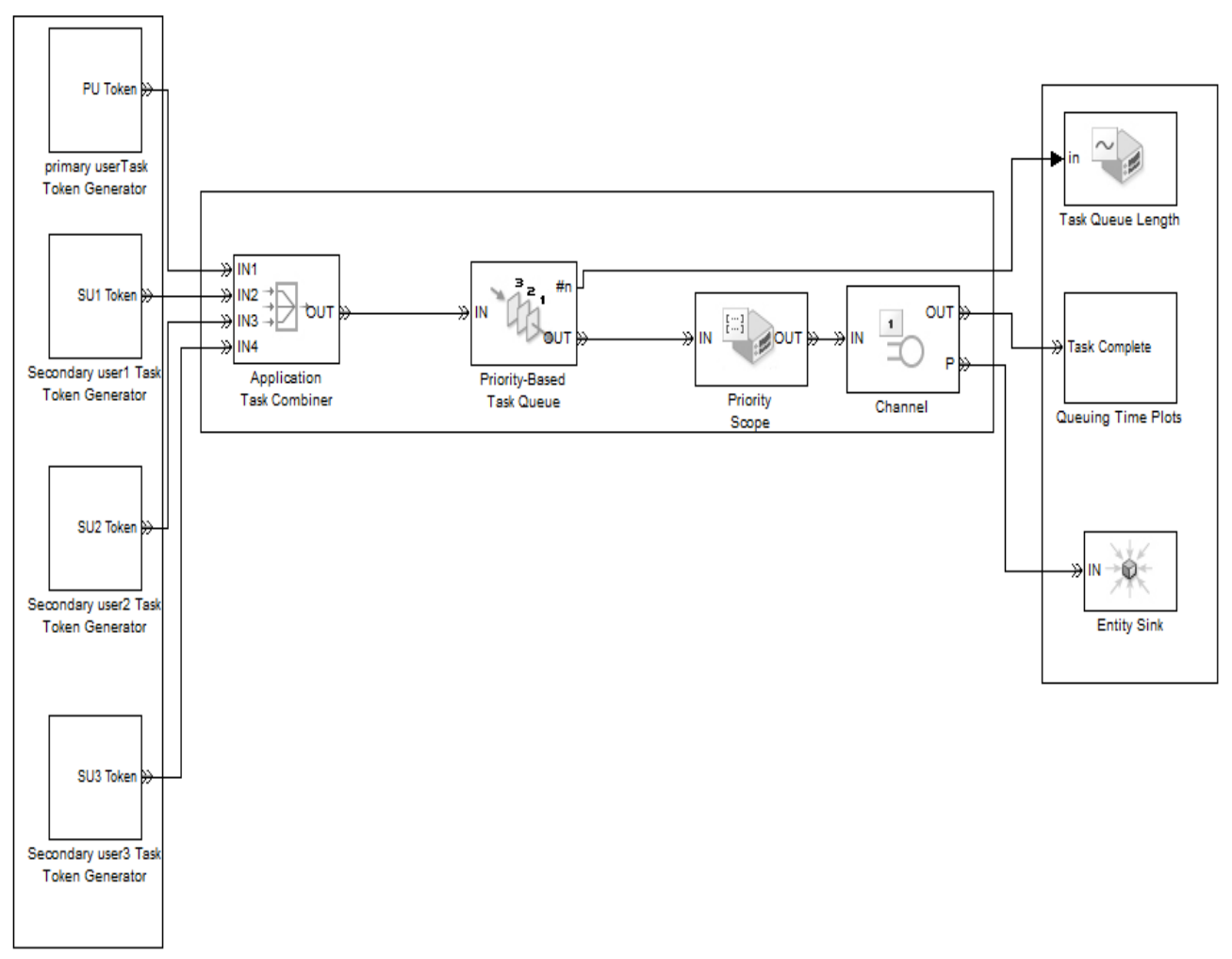

Fig. 1 The block diagram of the change policy .

\subsection{The second proposed model.}

This proposed model is shown in Fig. 2, applied the always stay policy with resuming the transmission. The low priority user stays on the same channel when the higher priority user arrives. The SU just pause its transmission during the high priority transmission and resumes afterward.

\section{Proposed approach operation steps:}

- Each token generator of the four users (PU, SU1, SU2, and SU3) generates the entities for each user. 


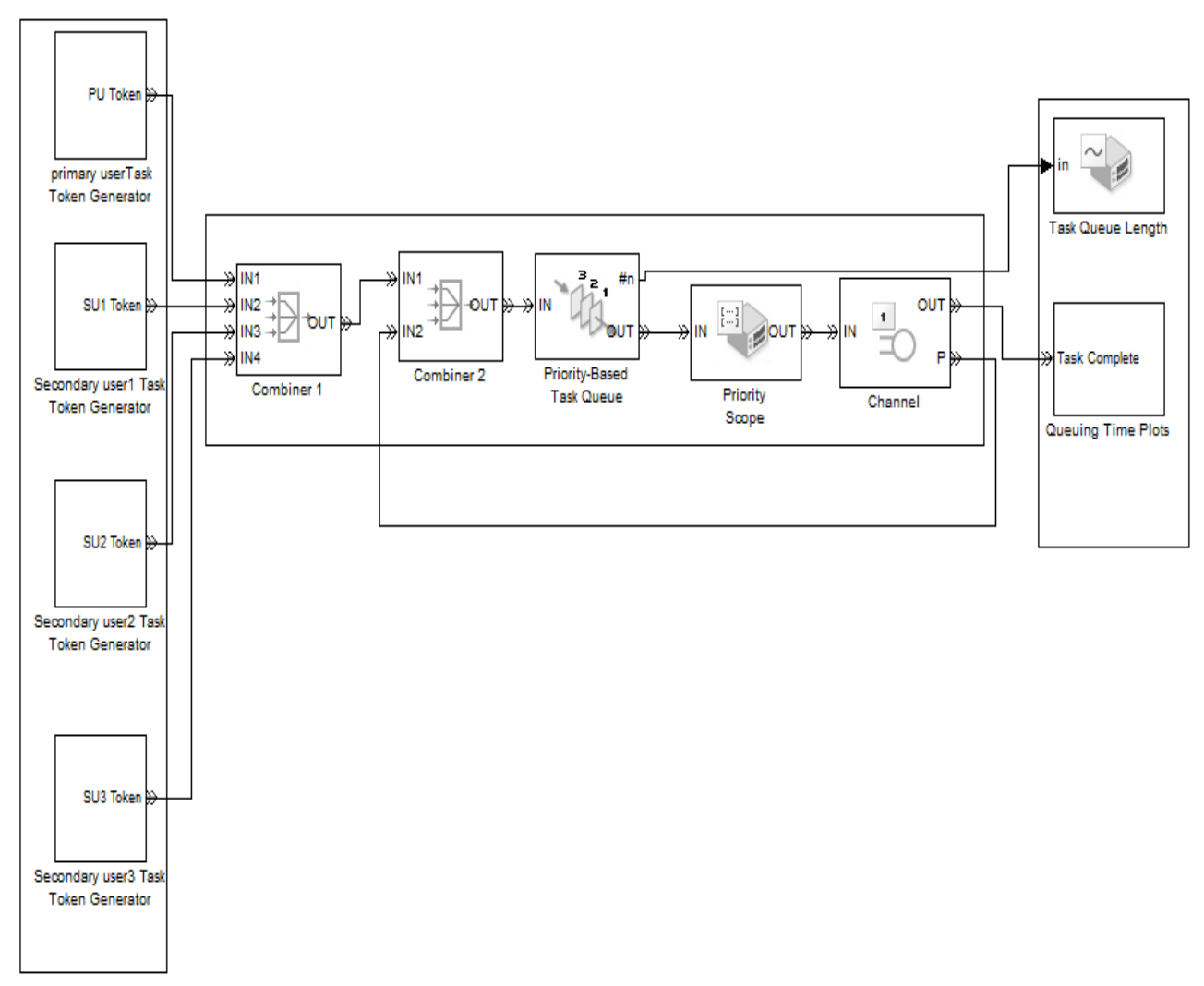

Fig. 2 The block diagram of the stay policy with resuming transmission.

- The tokens of four users are combined in the combiner 1 which merges the four input paths and outputs them through a single path to enter to combiner 2 .

- The combiner 2 combines the preempted entity from the channel which sampled by $\mathrm{P}$ port with the output of combiner 1, i.e, the preempted user always stay in the channel not change it.

- The output of combiner 2 enters as an input to priority-based task queue block which stores the entities of the users according to the priority. The capacity of this queue is assumed to be infinity. A scope of task queue 
length is used to show the length of the queue. The output of the prioritybased task queue block is shown by a priority scope.

- The output of priority scope input to the channel which serves the higher priority user. If a lower priority user transmits over the channel and the high priority user arrives, the transmission of lower priority user is paused and preempted through the $\mathrm{P}$ port and enters to combiner 2 and resumes its transmission after the higher priority user finishes its transmission, i.e, the low priority user stays in the channel not moves to another one and requires only the residual service time to finish its transmission.

- The output of the channel input to the task queue block to measure the total system time and the service time to compute the queuing delay for each user and plot it.

\subsection{The third proposed model.}

The third proposed model shown in Fig. 3, applied the always stay policy with restarting the transmission. The low priority user stays on the channel when the higher priority user arrives, but the difference in this policy, is that the user restarts its transmission from the beginning, i.e, the preempted user requires the same service time that required at the first time of transmission.

\section{Proposed approach operation steps:}

- As in the model of always stay policy with resuming transmission, four token generators are used: one for primary user token generator and three secondary user token generators.

- The generated entities from the four token generators combine by the combiner 1 which merges the four paths and output them through a single output port.

- The output of the combiner 1 combines with the preempted entities from the channel through $\mathrm{P}$ port in combiner 2 .

- Then the output passes to the priority-based task queue. To know the length of the queue, task queue length scope is used. A priority scope is also used to show the priority value for each task served in the channel. 
- The arranged entities according to its priority enter the channel to be served. The output of the channel enters to task complete block to calculate the queuing delay for each user. The other output port of the channel ( $\mathrm{P}$ port) which is the preempted entities reenters to the combiner 2 . In this system, the preempted user restarts its transmission not resumes it, therefore the required time for the preempted user is the whole service time, not the residual service time only.

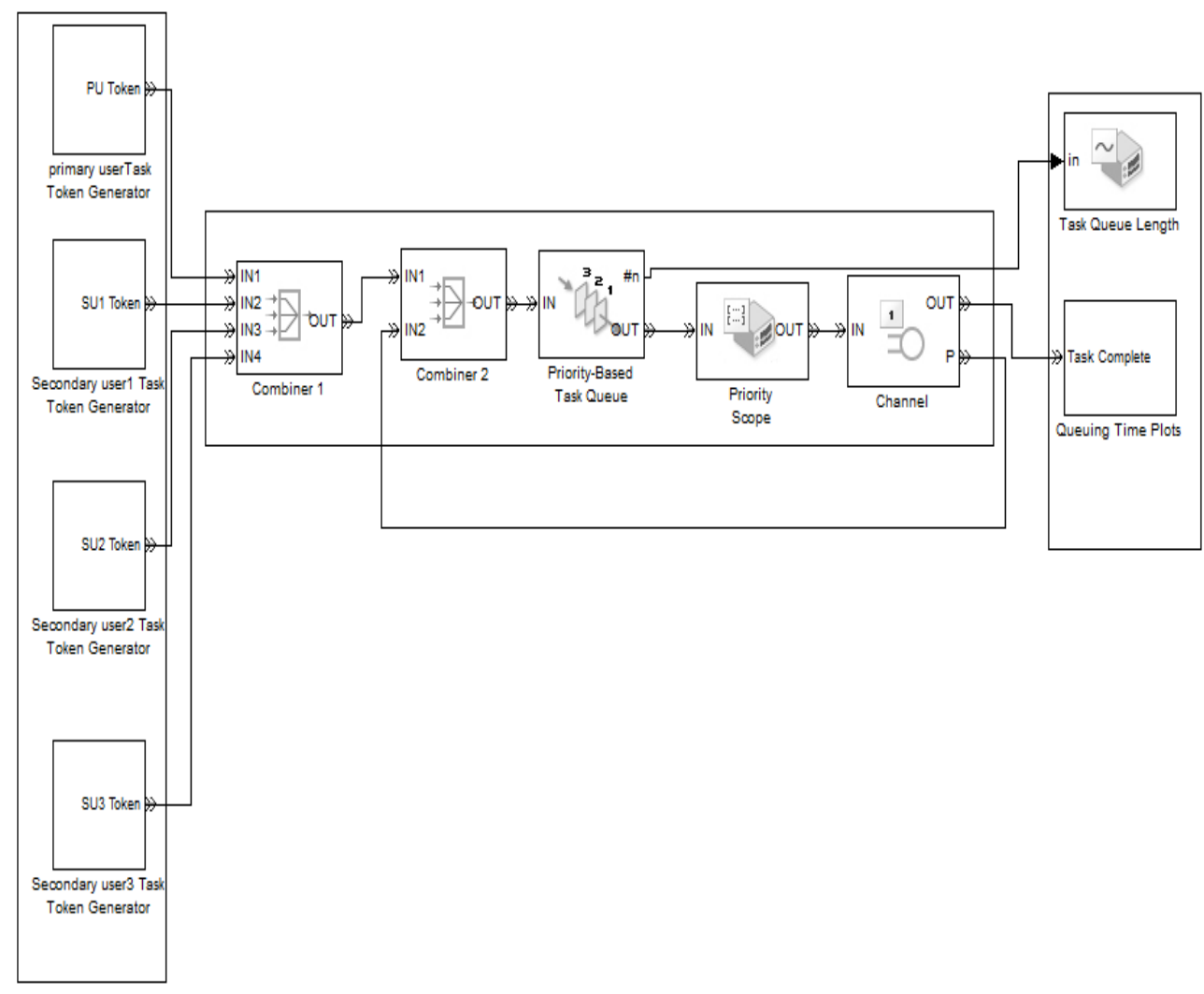

Fig.3 The block diagram of the always stay policy with restarting transmission. 


\section{Simulation results}

In the three proposed techniques, the simulation parameters are shown in Table 1:

\begin{tabular}{|l|l|}
\hline Number of PU & 1 \\
\hline Number of SU & 3 \\
\hline Distribution of PU signal & constant \\
\hline Distribution of SUs signal & exponential \\
\hline Capacity of priority queue & infinity \\
\hline Simulation time & $100 \mathrm{sec}$ \\
\hline
\end{tabular}

Table 1. the simulation parameters of the three proposed techniques.

\subsection{Processing results for the $1^{\text {st }}$ proposed model.}

Figure 4 indicates the priority value for the four users in the channel. The PU has the first priority to use the channel. At the time when the PU does not use the channel, the secondary users can use it by their priorities. The opportunity of using the channel decreases with increasing the priority. In Fig. 5, the queue length is plotted which is the number of tasks queued to be served in the channel. Figure 6 indicates the total system time for the PU which is the sum of the service time and the queuing delay time. The queuing delay for the PU can be obtained by subtraction the service time from the total system time for the PU as shown in Fig. 7. The delay for PU varies between 0 to $0.04 \mathrm{sec}$. Figure 8 shows the total system time for SU1. The service time $=0.36 \mathrm{sec}$ and the queuing delay is in the range from 0 to $0.81 \mathrm{sec}$ which is plotted in Fig. 9. For SU2, the total system time is indicated in Fig. 10. The service time equals to $0.3 \mathrm{sec}$ and the queuing delay time varies from 0: $2 \mathrm{sec}$ as shown in Fig. 11. The total system time for SU3 is illustrated in Fig. 12. Figure 13 shows the queuing delay for SU3. It reaches to $12.8 \mathrm{sec}$ as a maximum value. From the results, it is clear that the users that have lower priority, the opportunity for them to use the channel is low since the higher priority user may appear at any time. Consequently, the lower priority user must leave the channel and be preempted from the P port. Therefore, the SU3 rarely uses the channel. 


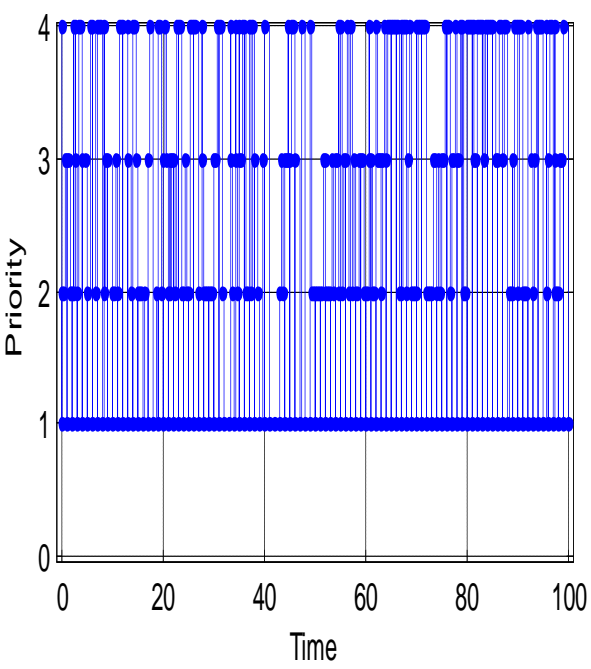

Fig.4 Priority value for tasks being served in the channel.

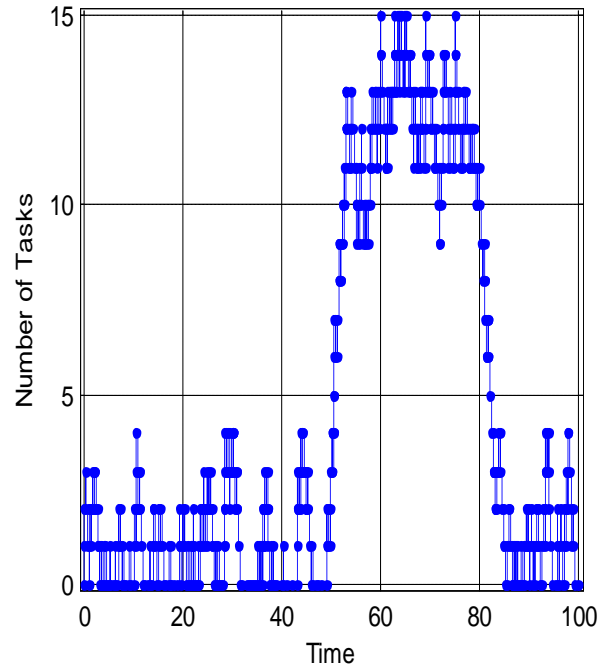

Fig.5 Queue length of the Priority-Based Task Queue.

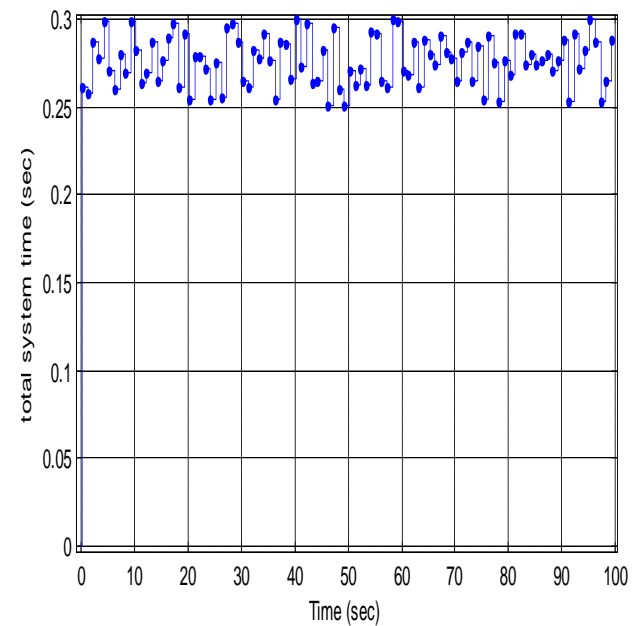

Fig.6 The total system time for PU tasks completed

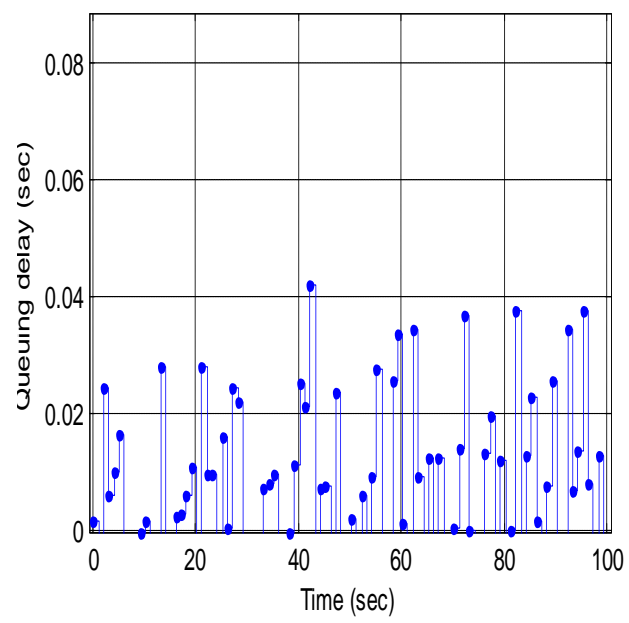

Fig.7Queuing delay for PU tasks completed. 

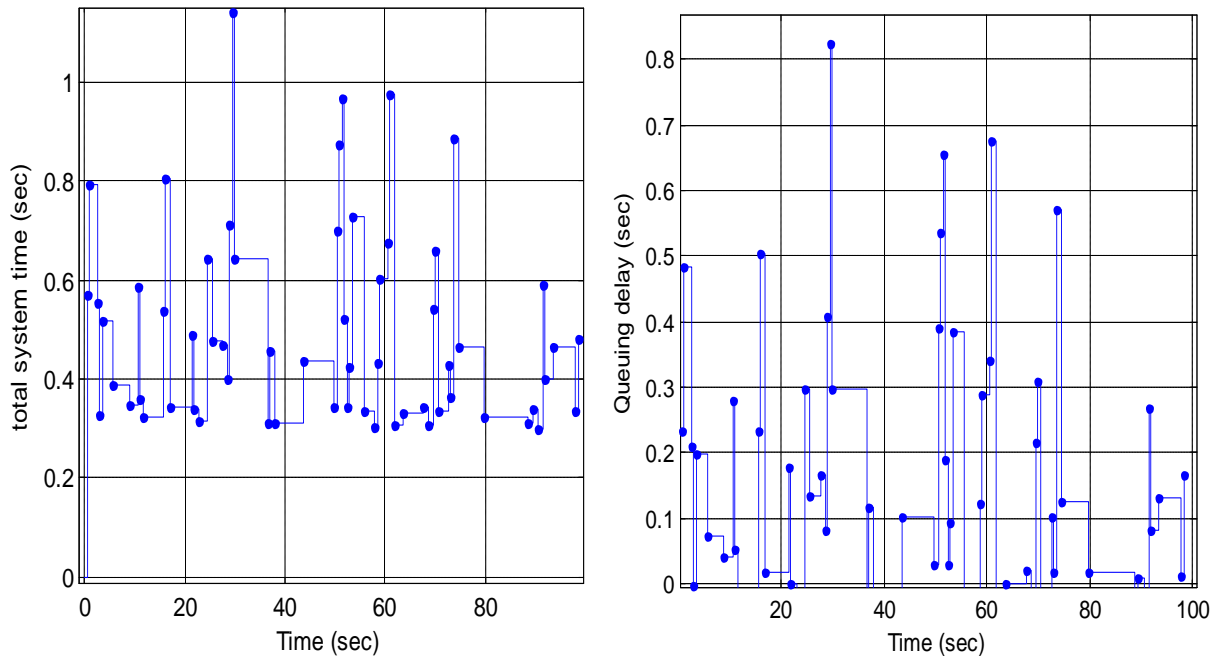

Fig.8The total system time for SU1 tasks completed. Fig.9Queuing delay for SU1 tasks completed.
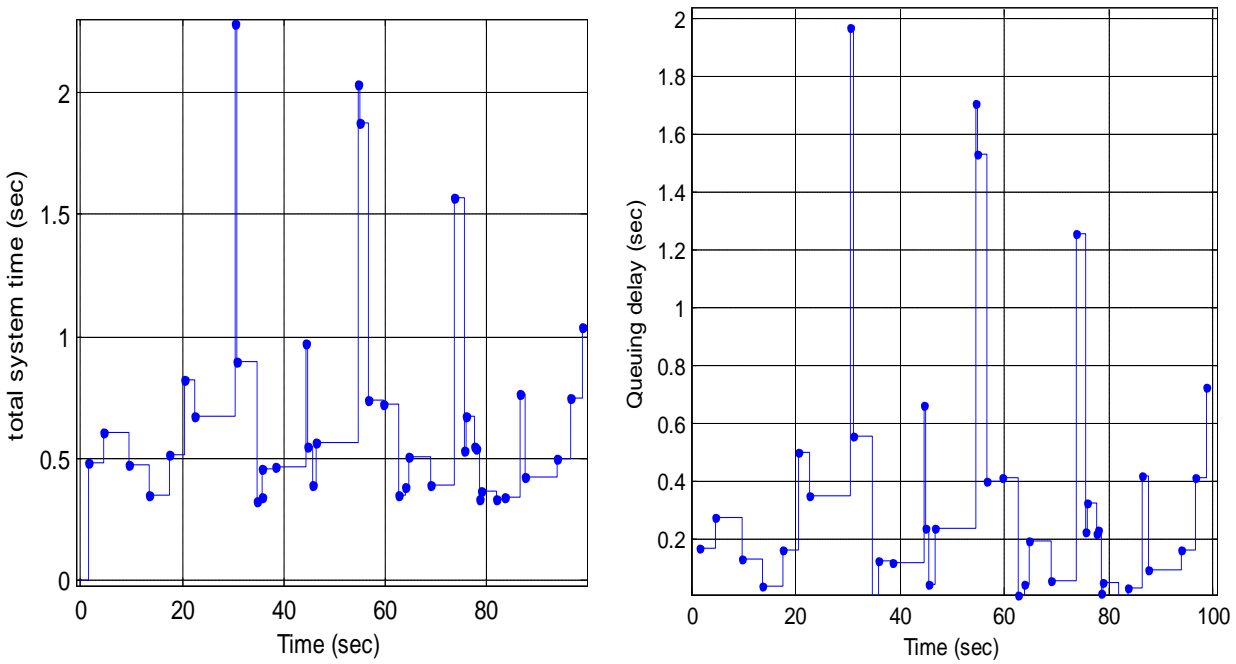

Fig.10The total system time for SU2 tasks completed. Fig.11Queuing delay for SU2 tasks completed. 


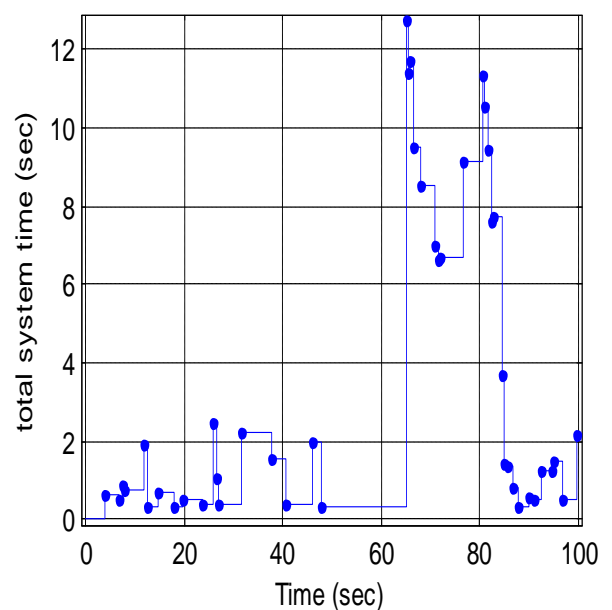

Fig.12The total system time for SU3 tasks completed.

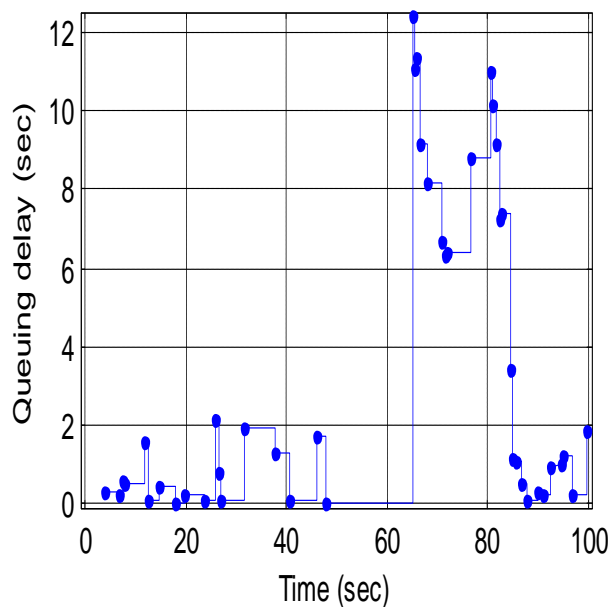

Fig.13Queuing delay for SU3 tasks completed.

\subsection{Processing results for the $2^{\text {nd }}$ proposed model.}

Figure 14 shows the priority value for the tasks of the four users being served in the channel. The PU has the highest priority; therefore it is the most user use the channel as shown in Fig. 14. The usage of the channel decreases with increasing the priority. This figure indicates that the opportunity of usage the channel from the SUs increases in this policy since the preempted entity does not leave the channel, but reenters the queue and waits to use the channel again. The queue length of the priority based task queue is plotted in Fig. 15. The number of tasks increases with increasing the simulation time. At time $=$ $100 \mathrm{sec}$, the number of tasks $=70$. The increase in the number of tasks compared with change policy returns to the always staying policy, i.e. the token still in the channel until it served. The total system time for PU and the queuing delay for its tasks are indicated in Fig. 16 and Fig. 17, respectively. It is clear that the same performance for the PU as in the change policy. That due to the PU must not be affected by the CRN. Therefore; it is not affected by the policy used in the CRN. The total system time for SU1 is plotted in Fig. 18. The service time $=0.4 \mathrm{sec}$ that can be concluded from this figure. The queuing delay is in the range from 0 to $2 \mathrm{sec}$ as shown in Fig. 19. With increasing the priority, the queuing delay is increasing. In Fig. 20, the total system for SU2 is plotted. It indicates that the service time $=0.4 \mathrm{sec}$ and the 
queuing delay in the range from 0 to $11 \mathrm{sec}$ as shown in Fig. 21. For the SU3, the total system is plotted in Fig. 22 which shows that the service time for $\mathrm{SU} 3=0.32 \mathrm{sec}$ and the queuing delay is in the range from 5.33 to $62 \mathrm{sec}$ which is the maximum value in this policy for all four users.
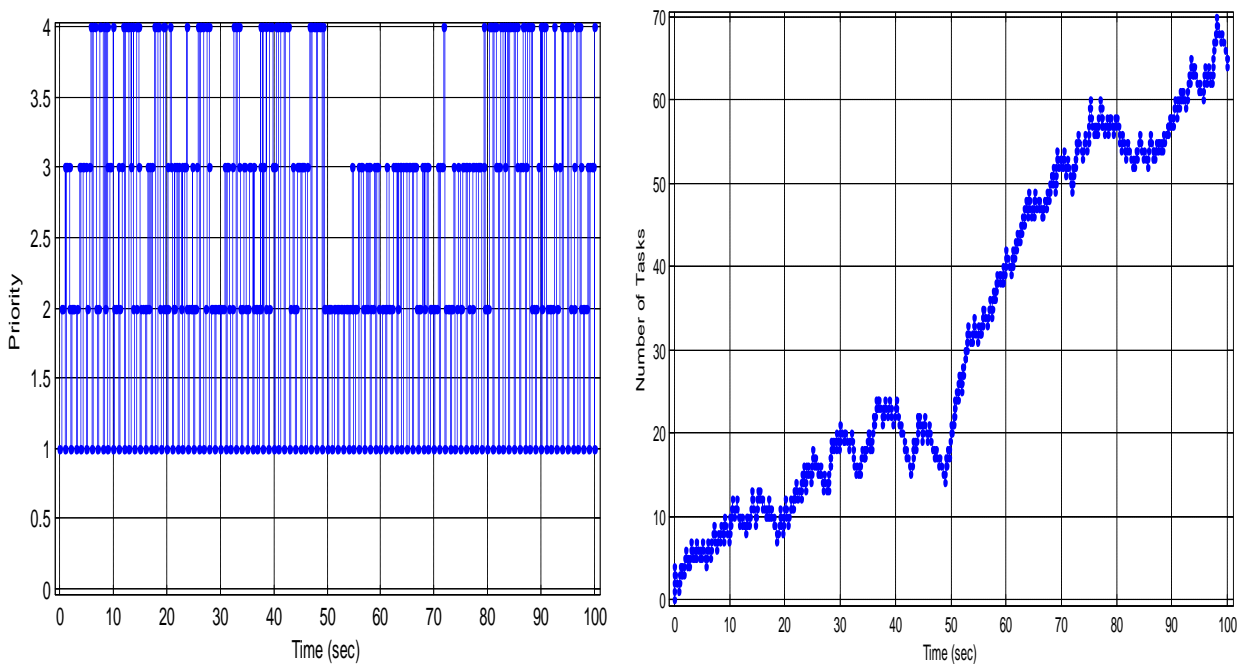

Fig.14 Priority value for tasks being served in the channel. Fig.15 Queue length of the Priority-Based Task Queue.
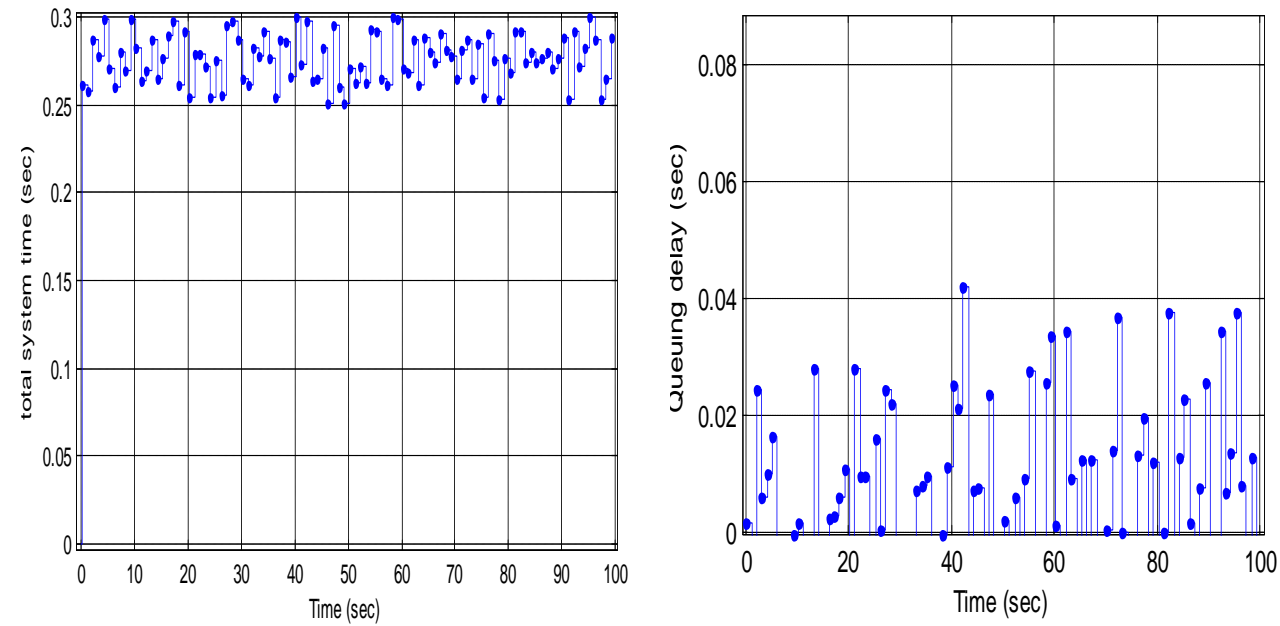

Fig.16 The total system time for PU tasks completed. Fig.17Queuing delay for PU tasks completed. 

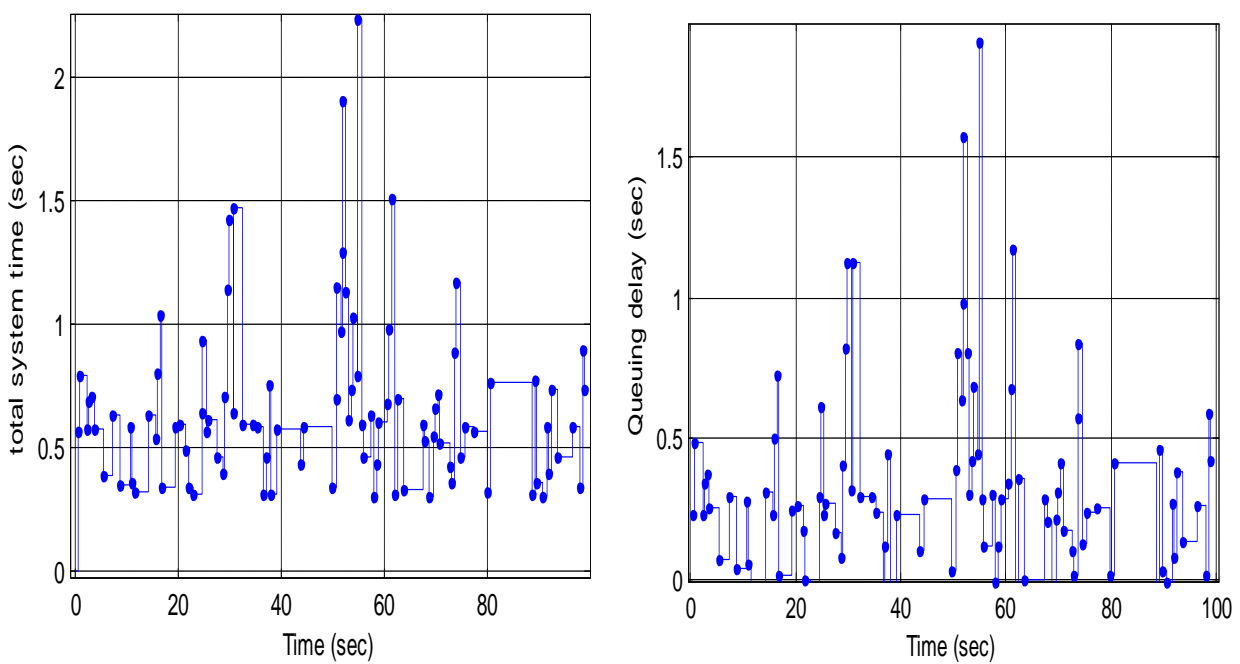

Fig.18The total system time for SU1 tasks completed. Fig.19Queuing delay for SU1 tasks completed.
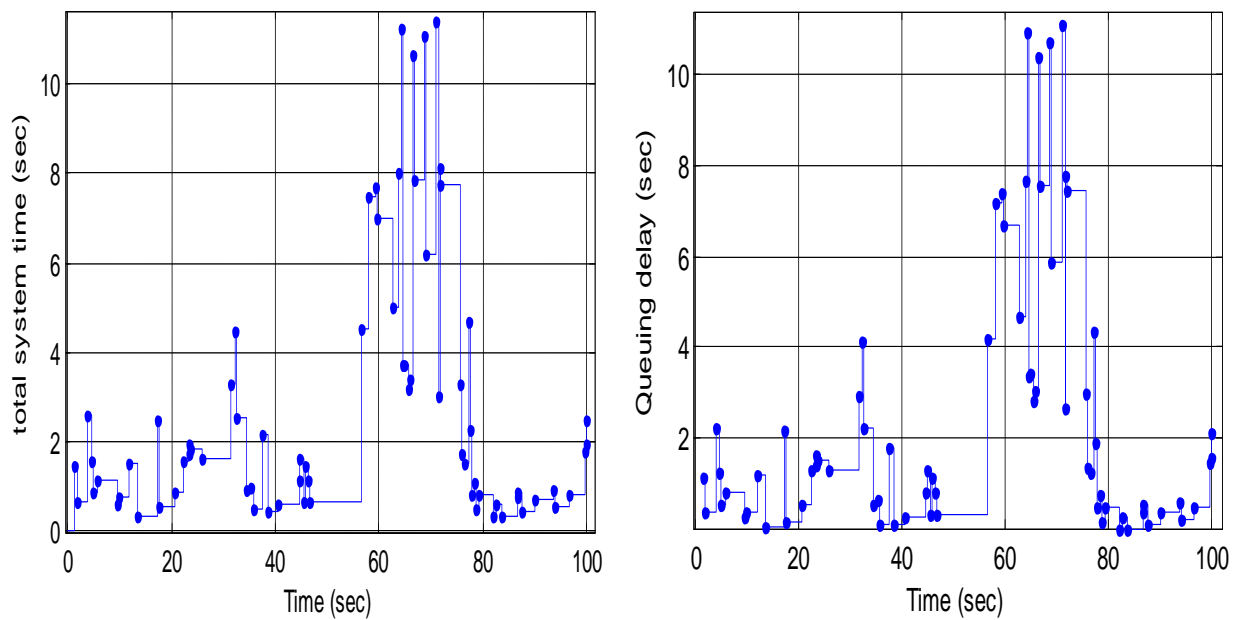

Fig.20The total system time for SU2 tasks completed. Fig.21Queuing delay for SU2 tasks completed. 

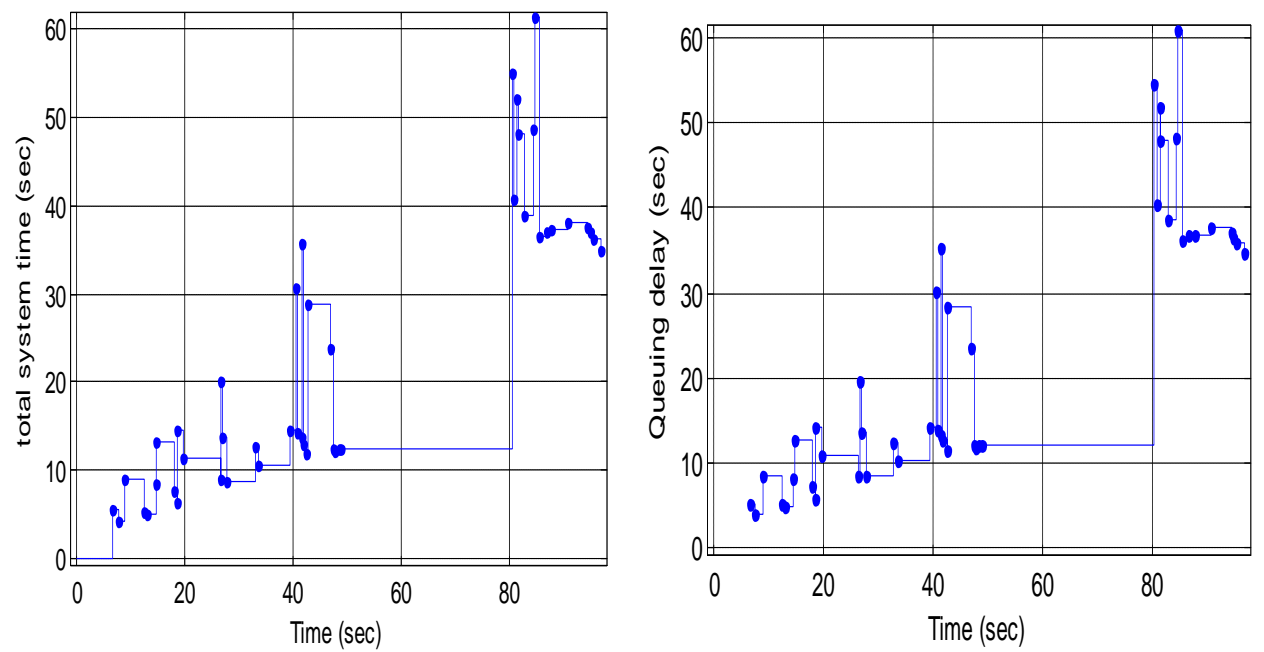

Fig.22The total system time for SU3 tasks completed. Fig.23Queuing delay for SU3 tasks completed.

\subsection{Processing results for the $3^{\text {rd }}$ proposed model.}

The preempted entities in this policy stay in the channel and restart the transmission from the beginning, i.e. it is not resuming it. Therefore; the entities take more time to complete its transmission. This leads to lessening the tasks for the SUs. Figure 24 shows the priority value for tasks being served in the channel. The number of the tasks being served in this policy is more than the change policy since the user stay in the channel until it be served, but it less than the tokens that served in the always staying with resuming transmission policy since the token not have to restart the transmission therefore; more time is saved for more users to use the channel. Figure 25 shows the queue length of the priority based task queue. It is obvious that the length of the queue reaches to 100 tasks which is high value if it compared with the other two policies. This due to restarting the transmission for each token not completed its transmission. By other words, if a token near to finish its transmission then a higher priority user appears, it enforces to leave the channel and stay in the channel and reenter the queue waiting for its role to use the channel. This means a lot of entities stay in the queue, therefore the queue length is longer than the other two policies. Figure 26 and Fig. 27 illustrate the total system time for the PU and the queuing delay for it which is the same at the three policies. For SU1, the total system 
time is indicated in Fig. 28. From this figure, the service time can be concluded to be $0.33 \mathrm{sec}$ and the queuing delay is $0: 2.8 \mathrm{sec}$ as shown in Fig. 29. The total system time for SU2 $=0.4 \mathrm{sec}$ and the queuing delay time $=0: 42$ sec as shown in Fig.30 and Fig.31 respectively. The opportunity of the entities of SU3 to complete its transmission is not high and if it occurred it will suffer from high delay.
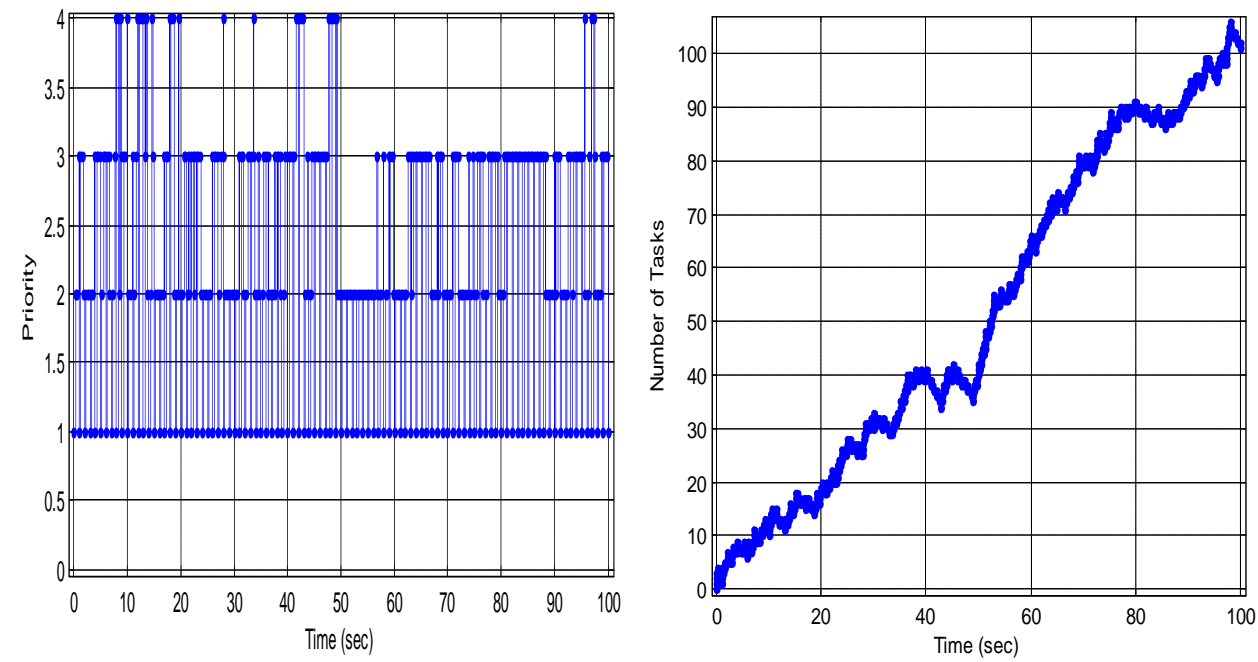

Fig.24 Priority value for tasks being served in the channel.

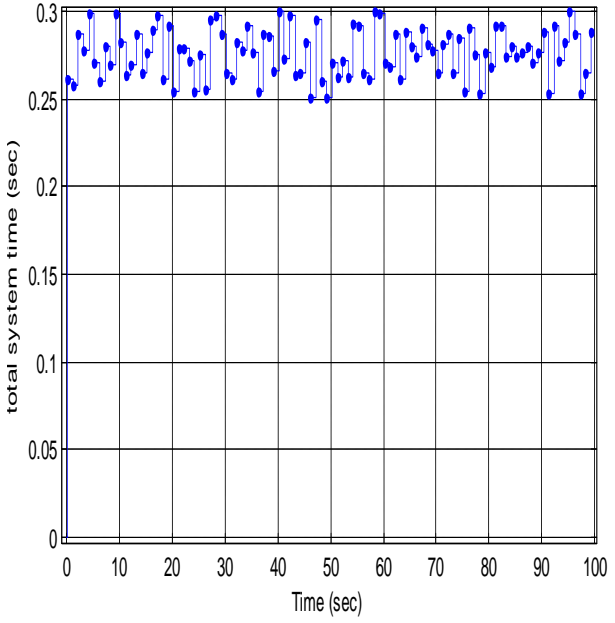

Fig.26 The total system time for PU tasks completed

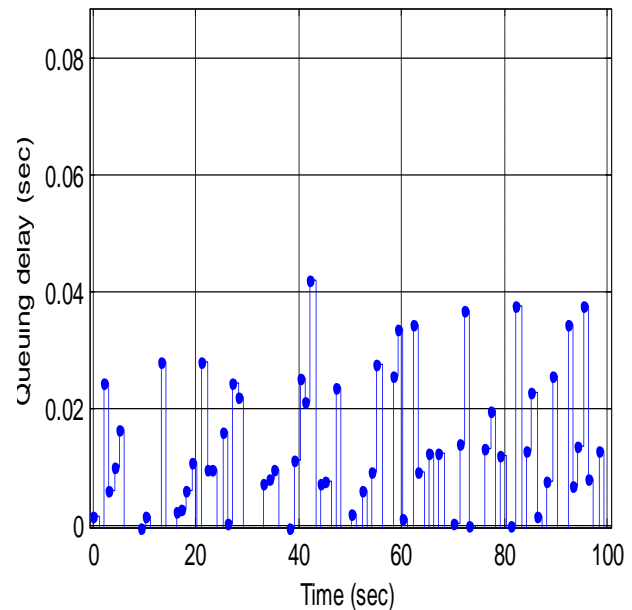

Fig.27Queuing delay for PU tasks completed. 

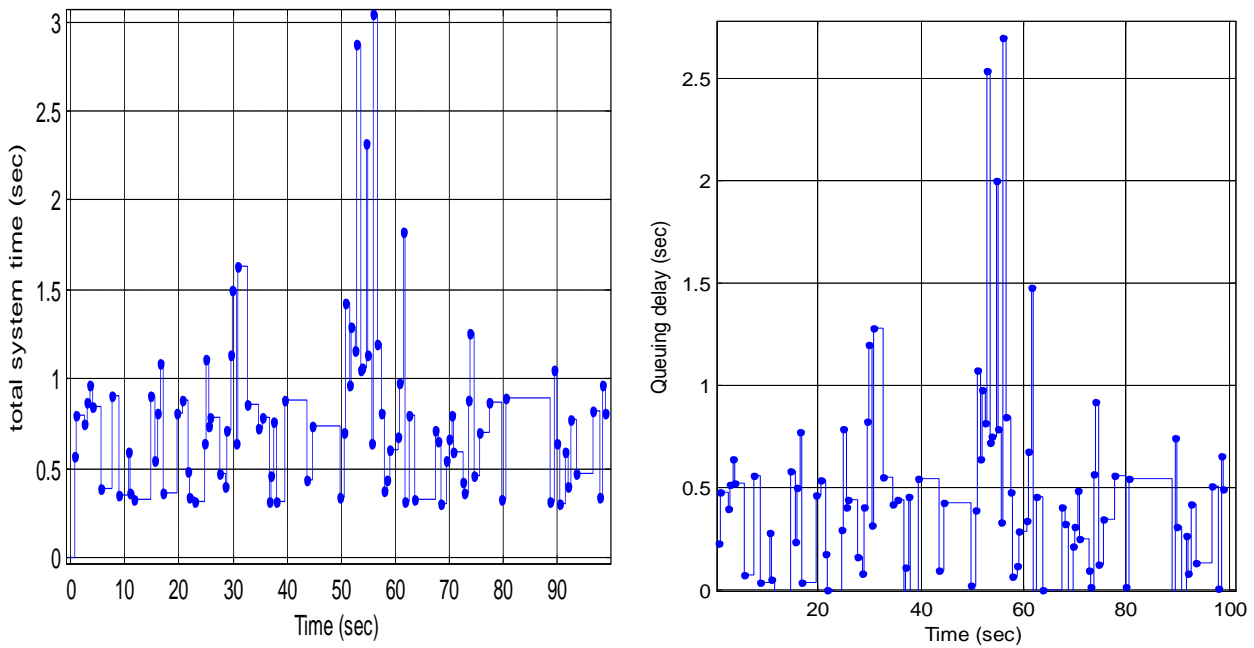

Fig.28The total system time for SU1 tasks completed. Fig.29Queuing delay for SU1 tasks completed.
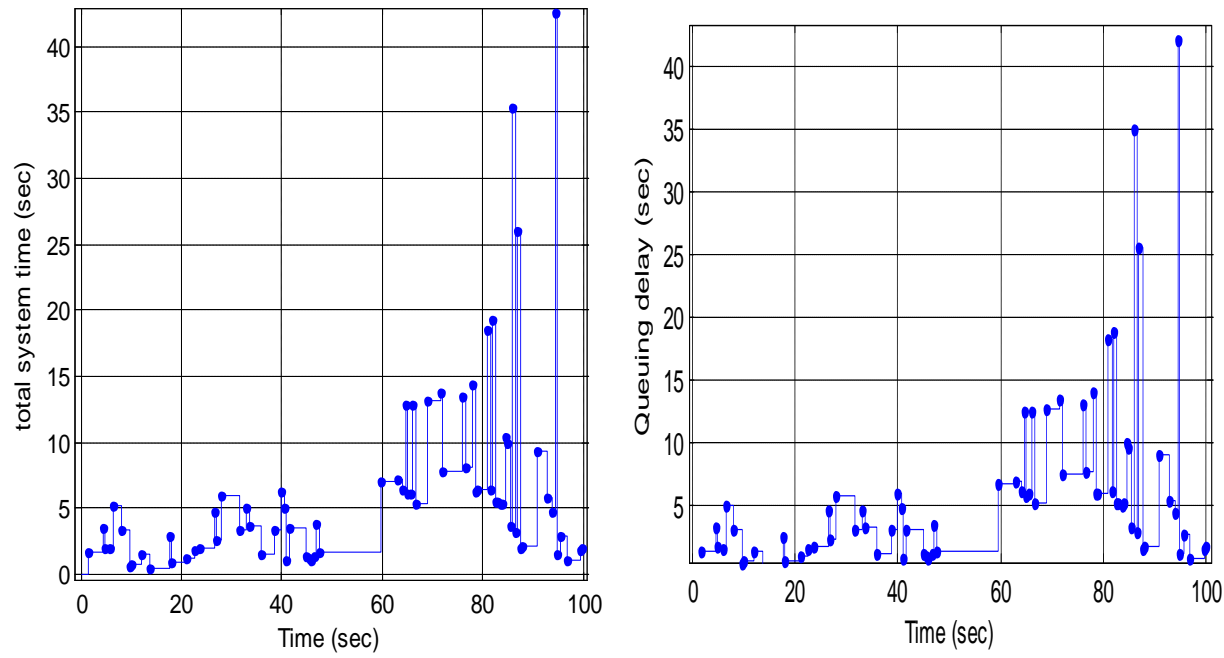

Fig.30The total system time for SU2 tasks completed. Fig.31Queuing delay for SU2 tasks completed. 

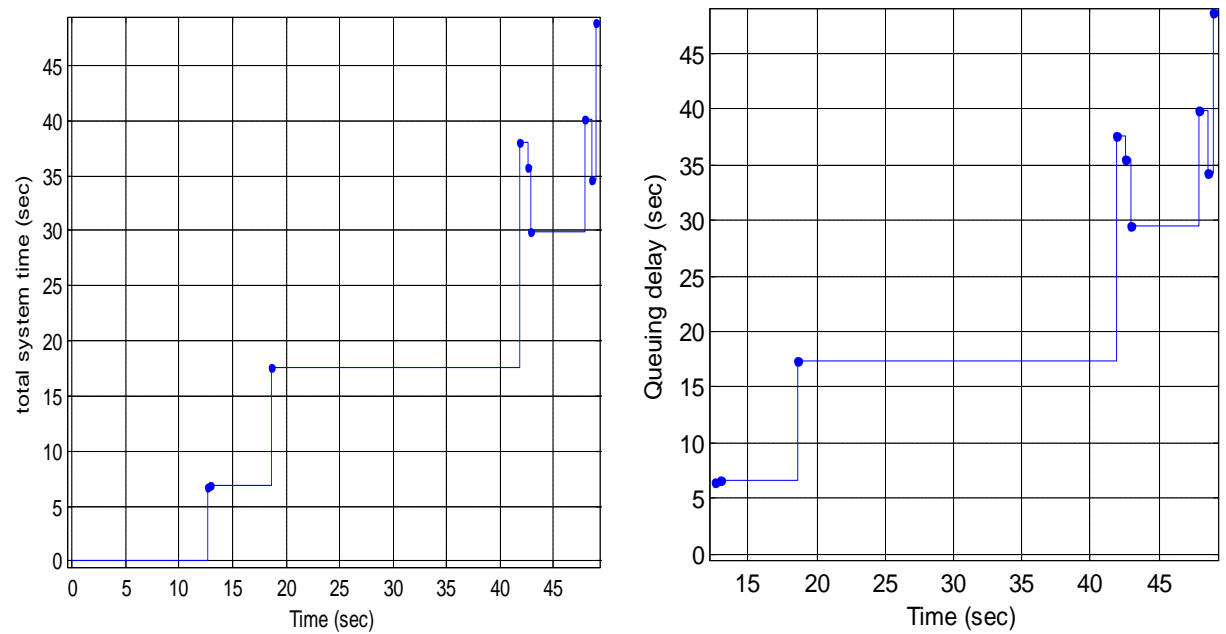

Fig.32The total system time for SU3 tasks completed. Fig.33Queuing delay for SU3 tasks completed.

The main applications of the proposed algorithms are summarized as follows:

- As shown in Table 2, the first proposed model has the minimum delay time for the secondary users; therefore it is suitable for the real-time applications.

- The second proposed model, stay with resuming transmission, has moderate values of delay, but the target channel selection problem is already solved by staying in the same channel. It is applied to characterize the connection-based spectrum handoff behaviors with multiple interruptions in multiple-channel CR networks [10]. It also used for having an optimal proactive target channel sequence selection algorithm to minimize the cumulative spectrum handoff delay in multiple-channel CR networks [8].

- The third proposed model, stay with restarting transmission, has high values of delay, but the target channel selection problem is already solved by staying in the same channel. And it has some applications using in the carrier sense multiple access collision detection (CSMA-CD) protocols to describe a fiber optical bus network, which connected a general finite number of stations [16]. 


\begin{tabular}{|l|l|l|l|}
\hline results & Change case & $\begin{array}{l}\text { Stay with } \\
\text { resuming }\end{array}$ & $\begin{array}{l}\text { Stay with } \\
\text { restarting }\end{array}$ \\
\hline $\begin{array}{l}\text { Max tasks in queue } \\
\text { length }\end{array}$ & 6 tasks & 70 tasks & 106 tasks \\
\hline $\begin{array}{l}\text { Max system time for } \\
\text { PU }\end{array}$ & $0.3 \mathrm{sec}$ & $0.3 \mathrm{sec}$ & $0.3 \mathrm{sec}$ \\
\hline $\begin{array}{l}\text { Max queuing delay for } \\
\text { PU }\end{array}$ & $0.04 \mathrm{sec}$ & $0.04 \mathrm{sec}$ & $0.04 \mathrm{sec}$ \\
\hline $\begin{array}{l}\text { Max system time for } \\
\text { SU1 }\end{array}$ & $1.14 \mathrm{sec}$ & $2.24 \mathrm{sec}$ & $3.05 \mathrm{sec}$ \\
\hline $\begin{array}{l}\text { Max queuing delay for } \\
\text { SU1 }\end{array}$ & $0.82 \mathrm{sec}$ & $1.91 \mathrm{sec}$ & $2.7 \mathrm{sec}$ \\
\hline $\begin{array}{l}\text { Max system time for } \\
\text { SU2 }\end{array}$ & $2.29 \mathrm{sec}$ & $11.41 \mathrm{sec}$ & $42.5 \mathrm{sec}$ \\
\hline $\begin{array}{l}\text { Max queuing delay for } \\
\text { SU2 }\end{array}$ & $1.97 \mathrm{sec}$ & $11 \mathrm{sec}$ & $42.2 \mathrm{sec}$ \\
\hline $\begin{array}{l}\text { Max system time for } \\
\text { SU3 }\end{array}$ & $1.3 \mathrm{sec}$ & $61.4 \mathrm{sec}$ & $48.9 \mathrm{sec}$ \\
\hline $\begin{array}{l}\text { Max queuing delay for } \\
\text { SU }\end{array}$ & $0.98 \mathrm{sec}$ & $61 \mathrm{sec}$ & $48.6 \mathrm{sec}$ \\
\hline
\end{tabular}

Table 2. the results for the three proposed techniques.

\section{Conclusions}

In this paper, M/ G/ 1 queuing model is used to characterize the behaviors of spectrum usage for three proposed techniques. Change policy, always staying 
with resuming transmission policy and always staying with restarting transmission policy are applied in CRN. For each user, the queuing delay and the opportunity of using the channel are studied in each policy. The change policy has the lowest values of delay for the three secondary users, but the target channel selection problem appears. Every policy in the three policies is suitable for a specific application. The three proposed models in this paper have been studying the delay performance for each user in CRN to determine the perfect application for each policy.

\section{References}

1- F. Akyildiz, W.-Y. Lee, M. C. Vuran, and S. Mohanty, Next generation/dynamic spectrum access/cognitive radio wireless network: A survey," in Computer Networks (Elsevier), November 2006, p. 56.

2- R.W. Thomas, L.A. Dasilva, A.B. Mackenzie, "Cognitive Networks", Proc. IEEE Int'l Symp. Dynamic Spectrum Access Networks (DySPAN), Nov. 2005.

3- S. Haykin, "Cognitive Radio: Brain-Empowered Wireless Communications", IEEE J. Selected Areas in Comm., vol. 23, no. 2, pp. 201-220, Feb. 2005.

4- L. Giupponi, and I. Pérez-Neira, "Fuzzy-Based Spectrum Handoff in Cognitive Radio Network," 3rd International Conference on Cognitive Radio Oriented Wireless Networks and Communication, CROWNCOM'2008, Singapore, May 2008.

5- Sh. Ali, M. Shokair, M. Dessouky, N. Messiha, H. El-Sayed, "Proposed Spectrum Handoff Algorithm in Cognitive Radio Networks Using Fuzzy Logic Control" CIIT, Networking and communication Engineering, vol.7, no. 5, 2015.

6- F. Sheikholeslami, M. Nasiri-Kenari, F. Ashtiani, "Optimal probabilistic initial and target channel selection for spectrum handoff in cognitive radio networks", IEEE Trans. Wireless Commun, vol. 14, pp. 570-584, 2015.

7- S. Zheng, X. Yang, S. Chen, S. Lou, "Target channel sequence selection scheme for proactive-decision spectrum handoff", IEEE Commun. Letters, vol.15, Dec. 2011, pp. 1332-1334.

8- L.-C. Wang, C.-W. Wang, C.-J. Chang," Optimal target channel sequence design for multiple spectrum handoffs in cognitive radio networks", IEEE Trans Commun., vol. 60, pp. 2444-2455, Sep. 2015.

9- L-C Wang and C-W. Wang, "Spectrum handoff for cognitive radio networks: reactive-sensing or proactive-sensing?" In: Proceedings of the IEEE 
international performance, computing and communication conference; pp. 343348.Dec. 2008.

10- L.-C. Wang and C.-W. Wang, "Modeling and Analysis for Spectrum Handoffs in Cognitive Radio Networks," IEEE Transactions on mobile computing, vol. 11, no. 9, Sep. 2012.

11- L.-C. Wang and C.-W. Wang, "Modeling and Analysis for Reactive-Decision Spectrum Handoff in Cognitive Radio Networks," Proc. IEEE Global Telecomm. Conf. (GlobeCom), 2010.

12- A. Lertsinsrubtavee, N. Malouch, and S. Fdida, "Controlling Spectrum Handoff with A Delay Requirement in Cognitive Radio Networks" $21^{\text {st }}$ international conference on computer communications and networks (ICCCN), pp1-8, Aug. 2012.

13- Y. Wu, F. Hu, S. Kumar, M. Guo, K. Bao, "Spectrum Handoffs with MixedPriority Queuing Model over Cognitive Radio Networks”, GlobalSIP, Dec. 2013.

14- L.-C. Wang, C.-W. Wang, and K.-T.Feng, "A queuing-theoretical framework for QoS-enhanced spectrum management in cognitive radio networks" IEEE Wireless Communications, vol. 18, no. 6, pp. 18-26, Dec. 2011.

15- X. Yang, X. Tan, L. Ye and Lin Ma, " Spectrum Handoffs Based on Preemptive Repeat Priority Queue in Cognitive Radio Networks", Sensors 2016, vol.16, no. 7, July 2016.

16- Jin, X. Min, G." Modeling and analysis of an integrated scheduling scheme with heterogeneous LRD and SRD traffic". IEEE Trans. Wireless Commun. Dec. 2013, 2598-2607. 


\section{ملخص البحث بالعربي}

دراسة أداء تأخير قائمة الانتظار في شبكة الراديو الإدراكية باستخدام طرق مختلفة لتسليم الطيف

$$
\begin{aligned}
& \text { م./ شيماء علي * ـ أ. د./ مني شقير * ـ أ.د./ معوض إبراهيم* أ.د./ ناجي مسيحه* }
\end{aligned}
$$

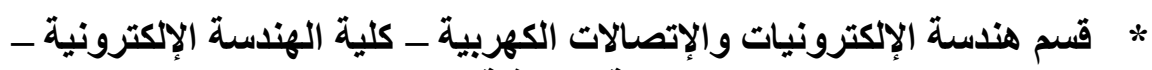

$$
\begin{aligned}
& \text { جامعة المنوفية. }
\end{aligned}
$$

من أهم شروط نقل الطيف في الثبكات الراديوية الإدراكية أن يكون هنائ تبديل سلس

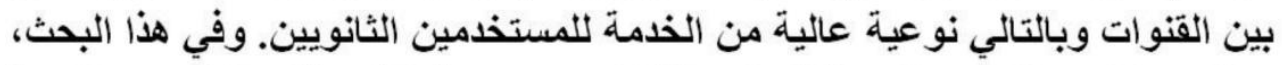

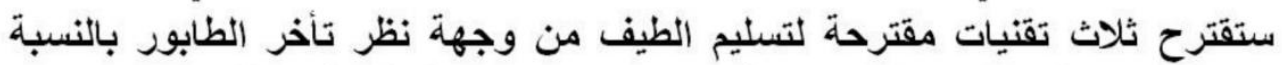

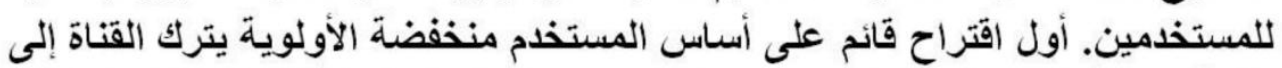

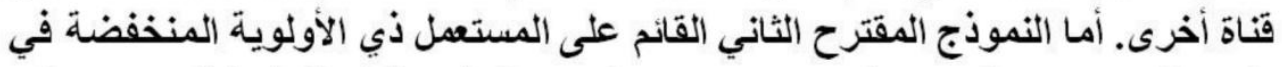

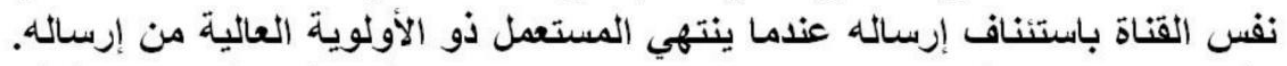

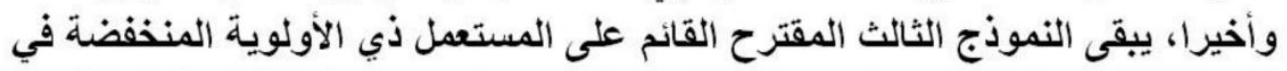

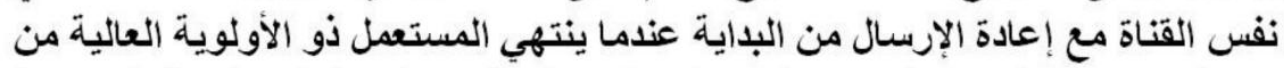

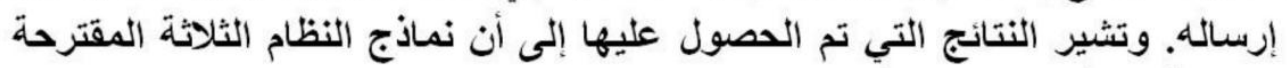

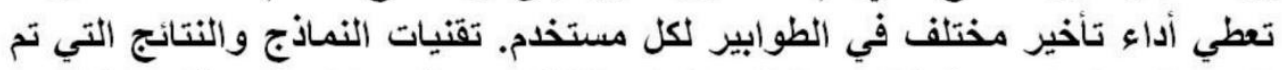

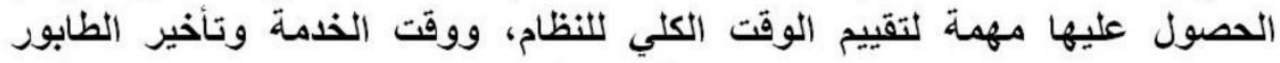

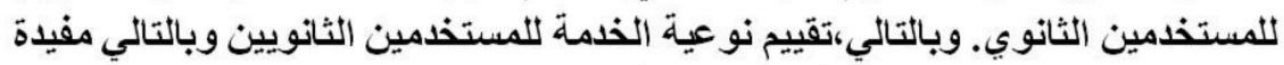
لتصميم الثبكة اللاسلكية المعرفية والتعديل فيها. 